(C) 1999 International Press

Adv. Theor. Math. Phys. 1 (1999) 83-90

\title{
Some Examples of Special Lagrangian Tori
}

\author{
Robert L. Bryant \\ Department of Mathematics \\ Duke University \\ Durham, NC 27708 \\ bryant@math. duke.edu
}

\section{Introduction}

The purpose of this note is to point out some very elementary examples of special Lagrangian tori in certain Calabi-Yau manifolds that occur as hypersurfaces in complex projective space. All of these are constructed as real slices of smooth hypersurfaces defined over the reals. This method of constructing special Lagrangian submanifolds is, of course, well known. What does not appear to be in the current mirror symmetry literature is an explicit description of such examples in which the special Lagrangian submanifold is a 3 -torus.

Some background. Let $M$ be a Ricci-flat Kähler $n$-manifold, with Kähler form $\omega \in \mathcal{A}_{+}^{1,1}(M)$ and a parallel holomorphic volume form $\Omega \in \mathcal{A}^{n, 0}(M)$, normalized so that

$$
\Omega \wedge \bar{\Omega}=\frac{2^{n}(-i)^{n^{2}}}{n !} \omega^{n} .
$$

The data $(\omega, \Omega)$ will be said to constitute a Calabi-Yau structure on $M$. Given any oriented $\omega$-Lagrangian submanifold $f: L \hookrightarrow M$ with induced volume form $d V_{L}$, there exists a smooth map $\lambda: L \rightarrow S^{1} \subset \mathbb{C}$ so that 


$$
f^{*}(\Omega)=\lambda d V_{L} .
$$

The submanifold $L$ is said to be special Lagrangian ${ }^{1}$ if $\lambda$ is identically 1 .

As Harvey and Lawson [HL] show, a compact special Lagrangian submanifold $L \subset M$ has minimal $\omega$-volume in its homology class. In fact, the real part of $\Omega$ is a calibration on $M$ and it calibrates the special Lagrangian submanifolds. Consequently, any smooth (or even $C^{1}$ ) special Lagrangian submanifold is real analytic in $M$.

McLean $[\mathrm{Mc}]$ showed that for a smooth special Lagrangian submanifold $L \subset M$, the moduli space of nearby special Lagrangian submanifolds is smooth and of dimension $b_{1}(L)$. He gave the following description: Let $\mathcal{H}^{1}(L) \subset \mathcal{A}^{1}(L)$ denote the vector space of harmonic 1-forms on $L$ (with respect to the induced metric). Then there exists an open neighborhood $\mathcal{U}$ of $0 \in \mathcal{H}^{1}(L)$ and a smooth (in fact, real analytic) mapping $Q: \mathcal{U} \rightarrow \mathcal{A}^{1}(L)$ that vanishes to second order at 0 so that, in a $C^{1}$ neighborhood of $f: L \hookrightarrow M$, any special Lagrangian normal graph is of the form $g_{\alpha}: L \rightarrow M$ where

$$
g_{\alpha}(x)=\exp _{f(x)}\left(i f^{\prime}\left((\alpha+Q(\alpha))_{x}^{\sharp}\right)\right),
$$

for a unique $\alpha \in \mathcal{H}^{1}(L)$. (Here, $\beta^{\sharp}$ denotes the vector in $T L$ dual to $\beta \in T^{*} L$ and multiplication by $i$ in $T M$ carries vectors tangent to $f(L)$ to vectors normal to $f(L)$.)

In particular, if $L$ is an $n$-torus and the induced metric on $L$ is such that there is a basis $\alpha_{1}, \ldots, \alpha_{n}$ of $\mathcal{H}^{1}(L)$ so that $\alpha_{1} \wedge \cdots \wedge \alpha_{n}$ is nowhere vanishing, then these normal graphs foliate a neighborhood of $L$ in $M$. Such a special Lagrangian torus is said to be foliating. Every special Lagrangian $n$-torus is foliating when $n=1$ or 2 , but this is not known to be the case in higher dimensions. $^{2}$

Conversely, if an open set $U \subset M$ has a foliation $\mathcal{L}$ by compact smooth special Lagrangian manifolds, then each leaf $L$ of $\mathcal{L}$ is diffeomorphic to the $n$-torus and has a basis $\alpha_{1}, \ldots, \alpha_{n}$ of $\mathcal{H}^{1}(L)$ so that $\alpha_{1} \wedge \cdots \wedge \alpha_{n}$ is nowhere vanishing.

In the last few years, these special Lagrangian foliations have become interesting in the theory of mirror symmetry, particularly after the paper

\footnotetext{
${ }^{1}$ Some authors require only that $\lambda$ be constant. For simplicity, I will not consider this (slight) generalization, though it is important for some purposes.

${ }^{2}$ This is trivial for $n=1$. For $n=2$ this follows from the fact a basis for the harmonic forms on $L$ is formed by the real and imaginary parts of a nonzero (and hence nonvanishing) holomorphic 1-form on $L$ (where $L$ is regarded as a complex curve of genus 1 via the conformal structure and orientation induced on $L$ by its inclusion into $M$ as a special Lagrangian submanifold).
} 
of Strominger, Yau, and Zaslow [SYZ]. Further work has been done on the geometry of moduli spaces of special Lagrangian tori and their augmentations by Hitchin [Hi1,2] and $\mathrm{Lu}[\mathrm{Lu}]$, among others. For example, in [Hi2], one sees how special Lagrangian foliations can be constructed entirely in the holomorphic category in cases where $n$ is even and $(M, \omega, \Omega)$ is actually hyperKähler.

In general, explicit examples of special Lagrangian tori seem to be difficult to construct. In the first place, the construction of Ricci-flat Kähler metrics itself relies on the celebrated theorem of Yau [Ya], which uses transcendental methods from nonlinear partial differential equations to construct a unique Ricci-flat Kähler form $\omega$ in the cohomology class of any Kähler form on a compact complex $M$ that is endowed with a holomorphic volume form $\Omega$. (By a Bochner argument, $\Omega$ is then parallel with respect to $\omega$.)

Many examples of compact complex Kähler manifolds with a holomorphic volume form are known, so Yau's theorem provides a rich source of examples of Ricci-flat Kähler manifolds. Perhaps the simplest examples (other than complex tori) are the smooth hypersurfaces of degree $n+1$ in $\mathbb{C P}^{n}$.

Given the data $(M, \omega, \Omega)$ it is usually a highly nontrivial matter to construct a special Lagrangian submanifold in $M$. One simple case where this can be done is when $(M, \omega, \Omega)$ possesses a real structure $c$, i.e., an involution $c: M \rightarrow M$, that satisfies $c^{*}(\omega)=-\omega$ and $c^{*} \Omega=\bar{\Omega}$. If the locus $L_{c} \subset M$ of fixed points of $c$ is nonempty, then it is a smooth manifold of real dimension $n$ that can be oriented so as to be a special Lagrangian submanifold of $M$. Moreover, since $c$ will necessarily preserve the underlying Kähler metric, $L_{c}$ is totally geodesic in $M$ with respect to this metric.

Of particular interest is the case of hypersurfaces $H \subset \mathbb{C P}^{n}$ of degree $n+1$ that are invariant under the anti-automorphism induced by a real structure $c: \mathbb{C}^{n+1} \rightarrow \mathbb{C}^{n+1}$, i.e., a complex antilinear involution, with fixed subspace $\mathbb{R}^{n+1} \subset \mathbb{C}^{n+1}$. In this case, the holomorphic volume form $\Omega$ on $H$ can be chosen to be antiïnvariant under the induced antiholomorphism $c_{H}$ : $H \rightarrow H$, i.e., so that $c_{H}^{*}(\Omega)=\bar{\Omega}$. If one chooses a class $\gamma \in H^{1,1}(H)$ that is representable by a Kähler form that also is antiïnvariant under this conjugation, ${ }^{3}$ then the uniqueness part of Yau's theorem implies that the unique Ricci-flat Kähler form $\omega$ on $M$ representing the class $\gamma$ must also be antiïnvariant under $c_{H}$. Consequently $c_{H}$ is a real structure on $(H, \omega, \Omega)$, implying that $L_{c}=H \cap \mathbb{R P}^{n}$ is a special Lagrangian submanifold.

Thus, one method of exhibiting a special Lagrangian torus is to find a $c$-invariant smooth hypersurface $H \subset \mathbb{C P}^{n}$ whose real locus $H \cap \mathbb{R} \mathbb{P}^{n}$ contains an $n$-torus as one of its components. In the following sections, I will point out

\footnotetext{
${ }^{3}$ These always exist. For example, take $\gamma$ to be the class of the pullback to $H$ of a $c$-invariant Fubini-Study metric on $\mathbb{C P}^{n}$.
} 
some simple examples of this for $n=2,3$, and 4 . Of course, the case $n=2$ is well-known and completely classical. Examples with $n=3$ are almost as classical. In fact, the possible topology of the real locus of a quartic surface in projective 3 -space was determined by Kharlamov [Kh], and there are several cases where this real locus contains a 2-torus component. Despite their simplicity, examples when $n=4$ seem not to have been pointed out in the mirror symmetry literature before. Of course it is this case that is of the most interest.

\section{The construction.}

The basic idea is straightforward. For any polynomial function $P$ : $\mathbb{C}^{n+1} \rightarrow \mathbb{C}$ that is homogeneous of degree $d \geq 1$, the equation $P=0$ defines a cone $C_{P} \subset \mathbb{C}^{n+1}$ and the equations $P=d P=0$ define a subcone $S_{P} \subset C_{P}$. Assume that $S_{P}$ is a proper subset of $C_{P}$. Let $\pi: \mathbb{C}^{n+1} \backslash\{\mathbf{0}\} \rightarrow \mathbb{C P}^{n}$ be the usual projection. Then the image $H_{P}=\pi\left(C_{P} \backslash\{\mathbf{0}\}\right)$ is a hypersurface in $\mathbb{C P}^{n}$ that is smooth away from the singular locus $\Sigma_{P}=\pi\left(S_{P} \backslash\{\mathbf{0}\}\right)$.

Let $\mathcal{P}$ denote the vector space consisting of polynomial functions $P$ : $\mathbb{C}^{n+1} \rightarrow \mathbb{C}$ that are homogeneous of degree $n+1$ and that satisfy $P \circ c=\bar{P}$ where $c: \mathbb{C}^{n+1} \rightarrow \mathbb{C}^{n+1}$ is the usual conjugation fixing $\mathbb{R}^{n+1} \subset \mathbb{C}^{n+1}$. This is a real vector space of dimension $\left(\begin{array}{c}2 n+1 \\ n\end{array}\right)$. For $P \in \mathcal{P}$, the loci $H_{P}$ and $\Sigma_{P}$ are invariant under the induced conjugation on $\mathbb{C P}^{n}$.

Let $\mathcal{O} \subset \mathcal{P}$ be the dense open subset consisting of those $P$ for which $\Sigma_{P}$ is empty. For $P \in \mathcal{O}$, endow $H_{P}$ with the unique Ricci-flat Kähler form $\omega_{P}$ whose Kähler class is dual to a hyperplane section. Then $\omega_{P}$ is antiïnvariant under conjugation and the holomorphic volume form $\Omega_{P}$ can be chosen so that $c^{*}\left(\Omega_{P}\right)=\bar{\Omega}_{P}$ and so that it satisfies the volume normalization needed to make its real part have comass one. This determines $\Omega_{P}$ up to a sign.

Let $\mathcal{T} \subset \mathcal{P}$ denote the open subset consisting of those $P \in \mathcal{P}$ for which the real slice $H_{P} \cap \mathbb{R} \mathbb{P}^{n}$ contains an $(n-1)$-torus $L \subset H_{P} \cap \mathbb{R} \mathbb{P}^{n}$ that is disjoint from $\Sigma_{P}$. In the following sections, I will show that $\mathcal{T}$ is nonempty for $n=2$, 3 , and 4 by producing explicit examples. I do not know whether or not $\mathcal{T}$ is nonempty for higher $n$.

When $\mathcal{T}$ is nonempty, it follows that $\mathcal{O} \cap \mathcal{T}$ is nonempty (and open). For $P \in \mathcal{O} \cap \mathcal{T}$, the smooth hypersurface $H_{P} \subset \mathbb{C P}^{n}$ contains an $(n-1)$-torus as a component of its real locus. Such an $(n-1)$-torus is special Lagrangian in the Calabi-Yau structure $\left(H_{P}, \omega_{P}, \Omega_{P}\right)$. 


\section{Cubic Curves in $\mathbb{C P}^{2}$}

This section is included for the sake of completeness and for comparison with the cases where $n=3$ and 4 . Any smooth cubic curve defined over $\mathbb{R}$ is projectively equivalent to $H_{P}$ where

$$
P=X_{0}{ }^{3}+X_{1}{ }^{3}+X_{2}{ }^{3}-3 \sigma X_{0} X_{1} X_{2}
$$

for some real number $\sigma \neq 1$. The curve $H_{P}$ has two real components when $\sigma>1$ and one real component when $\sigma<1$. In either case, there is always exactly one odd component ${ }^{4}$ and it contains the three real flexes $f_{1}=$ $[0,1,-1], f_{2}=[-1,0,1]$, and $f_{3}=[1,-1,0]$.

When $\sigma=1$, the curve $H_{P}$ is singular, being the union of three lines, one of which is real, namely $H_{L}$, where $L=X_{0}+X_{1}+X_{2}$. Note that the singular locus $\Sigma_{P}$ consists of three points, one of which is real, but which does not lie on $H_{L}$.

Thus, $P$ lies in $\mathcal{T}$ for any finite $\sigma$. (When $\sigma=\infty$, the real locus consists of three real non-concurrent lines and hence has no smooth component.) Note also that $\mathcal{O} \cap \mathcal{T}$ has two components.

\section{$3 \quad$ Quartic Surfaces in $\mathbb{C P}^{3}$}

The topology of the real locus of a quartic surface in projective 3-space was determined in the 1970s. For a survey of these results, see Kharlamov [Kh]. However, it is easy to construct elements of $\mathcal{T}$ directly. Here are a few simple examples, generalizing both the 'odd component' and the 'even component' case of curves.

As a first example, consider

$$
P=X_{0}{ }^{4}+X_{1}^{4}-X_{2}^{4}-X_{3}^{4} .
$$

This is a nonsingular Fermat-type quartic. The real locus is

$$
H_{P} \cap \mathbb{R}^{3}=\left\{[a, b, u, v] \in \mathbb{R} \mathbb{P}^{3} \mid a^{4}+b^{4}=u^{4}+v^{4}=1\right\} .
$$

Since the curve $C \subset \mathbb{R}^{2}$ defined by $x^{4}+y^{4}=1$ is diffeomorphic to the circle, $H_{P} \cap \mathbb{R}^{3}$ is diffeomorphic to $(C \times C) / \sim$ where $((a, b),(u, v)) \sim$ $((-a,-b),(-u,-v))$. This is manifestly a 2 -torus. Consequently, $P$ lies in $\mathcal{T}$.

\footnotetext{
${ }^{4} \mathrm{~A}$ simple closed curve in $\mathbb{R} \mathbb{P}^{2}$ is odd if it generates $H_{1}\left(\mathbb{R} \mathbb{P}^{2}, \mathbb{Z}_{2}\right) \simeq \mathbb{Z}_{2}$, otherwise it is even.
} 
Note that $\mathbb{R} \mathbb{P}^{3} \cap H_{P}$ is like the odd component of an elliptic curve: It is not contractible in $\mathbb{R P}^{3}$ because its inverse image under the double cover $S^{3} \rightarrow$ $\mathbb{R} \mathbb{P}^{3}$ is a nontrivial double cover.

As a second example, take

$$
R=\left(X_{0}^{2}+X_{1}^{2}-r_{1}\left(X_{2}^{2}+X_{3}^{2}\right)\right)\left(X_{0}^{2}+X_{1}{ }^{2}-r_{2}\left(X_{2}^{2}+X_{3}^{2}\right)\right)
$$

where $0<r_{1}<r_{2}$. In this case, the real locus $\mathbb{R}^{3} \cap H_{R}$ consists of two disjoint 2-tori, neither of which is contractible in $\mathbb{R}^{3}$. The singular locus $\Sigma_{R}$ consists of the four lines

$$
L_{m, n}=\left\{\left[a,(-1)^{m} i a, b,(-1)^{n} i b\right] \mid[a, b] \in \mathbb{C P}^{1}\right\}, \quad m, n \in\{0,1\} .
$$

and these have no real points.

The third example is more like the even component of a real elliptic curve. In $\mathbb{R}^{3}$, consider the circle $C$ defined by the equations

$$
x_{1}^{2}+x_{2}^{2}-1=x_{3}=0 .
$$

This is the minimum locus of the quartic polynomial $q_{0}$ defined by

$$
q_{0}=\left(x_{1}^{2}+x_{2}^{2}-1\right)^{2}+x_{3}^{4} .
$$

The critical locus of $q_{0}$ in $\mathbb{R}^{3}$ is the circle $C$ plus the origin $O=(0,0,0)$. Consequently, the regular values of $q_{0}$ are all real numbers other than 0 and 1.

Let $\epsilon$ be a real number satisfying $0<\epsilon<1$ and consider the quartic polynomial $q_{\epsilon}=q_{0}-\epsilon$. Its zero locus in $\mathbb{R}^{3}$ is smooth and is the boundary of the region $R_{\epsilon}=q_{0}^{-1}([0, \epsilon])$, which retracts onto the circle $C$. Consequently, the zero locus of $q_{\epsilon}$ is diffeomorphic to a torus.

Now consider the homogeneous quartic $Q$ defined by

$$
\begin{aligned}
Q & =\left(X_{1}^{2}+X_{2}^{2}-X_{0}^{2}\right)^{2}+X_{3}^{4}-\epsilon X_{0}^{4} \\
& =X_{0}^{4} q_{\epsilon}\left(X_{1} / X_{0}, X_{2} / X_{0}, X_{3} / X_{0}\right)
\end{aligned}
$$

The singular locus $\Sigma_{Q}$ consists of two nonreal points $[0,1, \pm i, 0]$.

Since $Q=X_{0}=0$ on $\mathbb{R}^{4}$ only at the origin, the real slice $H_{Q} \cap \mathbb{R P}^{3}$ is just

$$
T=\left\{\left[1, X_{1}, X_{2}, X_{3}\right] \mid q_{\epsilon}\left(X_{1}, X_{2}, X_{3}\right)=0\right\}
$$


and hence is diffeomorphic to a torus. Thus $Q$ lies in $\mathcal{T}$.

Note that $T$ misses a linear $\mathbb{R} \mathbb{P}^{2}$ in $\mathbb{R}^{3}$ and consequently is contractible in $\mathbb{R P}^{3}$. Since $H_{Q} \cap \mathbb{R P}^{3}$ is not homotopic to $H_{P} \cap \mathbb{R P}^{3}$, it follows that $P$ and $Q$ lie in different components of $\mathcal{T}$. By these three examples, it follows that $\mathcal{O} \cap \mathcal{T}$ has at least three components.

\section{Quintic 3-folds in $\mathbb{C P}^{4}$}

In $\mathbb{R}^{4}$, consider the locus $T$ defined by the equations

$$
\begin{aligned}
& x_{1}{ }^{2}+x_{2}{ }^{2}=1 \\
& x_{3}{ }^{2}+x_{4}{ }^{2}=1
\end{aligned}
$$

This is a 2-torus (it is the Clifford torus, up to scale). Because it is cut out by two independent equations, the normal bundle $N$ of $T$ in $\mathbb{R}^{4}$ is trivial, implying that its unit circle bundle $L \subset N$ is diffeomorphic to the 3 -torus.

Consider the quartic function

$$
q=\left(x_{1}^{2}+x_{2}{ }^{2}-1\right)^{2}+\left(x_{3}{ }^{2}+x_{4}{ }^{2}-1\right)^{2} .
$$

Then $q(x) \geq 0$, with equality if and only if $x$ lies in $T$. The critical values of $q$ are 0,1 , and 2 .

Thus, for $\epsilon$ satisfying $0<\epsilon<1$, the hypersurface $T_{\epsilon} \subset \mathbb{R}^{4}$ defined by $q(x)-\epsilon^{2}=0$ is the boundary of a compact domain that has $T$ as a deformation retract. In fact, by Morse theory, $T_{\epsilon}$ is diffeomorphic to $L \subset N$, which, as has been remarked, is a 3 -torus.

Now consider the (reducible) quintic polynomial $P$ defined by

$$
\begin{aligned}
P & =X_{0}^{5}\left(q\left(X_{1} / X_{0}, X_{2} / X_{0}, X_{3} / X_{0}, X_{4} / X_{0}\right)-\epsilon^{2}\right) \\
& =X_{0}\left[\left(X_{1}^{2}+X_{2}^{2}-X_{0}^{2}\right)^{2}+\left(X_{3}^{2}+X_{4}^{2}-X_{0}^{2}\right)^{2}-\epsilon^{2} X_{0}^{4}\right] \\
& =X_{0} Q, \quad(Q \text { is irreducible }) .
\end{aligned}
$$

The quintic hypersurface $H_{P} \subset \mathbb{C P}^{4}$ is the union of the hyperplane $H_{X_{0}}$ and the quartic hypersurface $H_{Q}$.

The singular locus $\Sigma_{Q}$ is a union of four lines

$$
L_{m, n}=\left\{\left[0, a,(-1)^{m} i a, b,(-1)^{n} i b\right] \mid[a, b] \in \mathbb{C P}^{1}\right\}, \quad m, n \in\{0,1\} .
$$


None of these lines have real points.

The intersection $H_{X_{0}} \cap H_{Q}$ is a union of two quadric surfaces

$$
S_{ \pm}=\left\{\left[0, X_{1}, X_{2}, X_{3}, X_{4}\right] \mid\left(X_{1}^{2}+X_{2}^{2}\right) \pm i\left(X_{3}^{2}+X_{4}^{2}\right)=0\right\} .
$$

Neither of these surfaces has any real points. Note that $S_{+} \cap S_{-}$consists of the four lines $L_{m, n}$. Consequently, $\Sigma_{P}=S_{+} \cup S_{-}$.

Thus, the real slice $H_{P} \cap \mathbb{R P}^{4}$ is smooth and is the disjoint union of $H_{X_{0}} \cap$ $\mathbb{R} \mathbb{P}^{4} \simeq \mathbb{R}^{3}$ and $H_{Q} \cap \mathbb{R} \mathbb{P}^{4} \simeq T_{\epsilon}$, which is diffeomorphic to the 3 -torus.

Thus, $P$ lies in $\mathcal{T}$, which is thereby shown to be nonempty. Consequently, $\mathcal{O} \cap \mathcal{T}$ is nonempty, thus proving that there are smooth (and hence irreducible) quintic hypersurfaces in $\mathbb{C P}^{4}$ whose real locus contains a torus as a component.

\section{References}

[1] N. Hitchin, The moduli space of special Lagrangian submanifolds, dgga/9711002.

[2] N. Hitchin, The moduli space of complex Lagrangian submanifolds, dg$\mathrm{ga} / 9901069$.

[3] F. Harvey and H. Lawson, Calibrated Geometries, Acta. Math. 148 (1982), 47-157. MR 85i:53058.

[4] V. Kharlamov, On the classification of nonsingular surfaces of degree 4 in $\mathbb{R} \mathbb{P}^{3}$ with respect to rigid isotopies. (Russian) Funktsional. Anal. i Prilozhen. 18 (1984), 49-56. (English translation in Functional Anal. Appl. 18 (1984), 39-45.) MR 85m:14034.

[5] P. Lu, Special Lagrangian Tori on a Borcea-Voisin Threefold, dgga/9902063.

[6] R. MCLean, Deformations of calibrated submanifolds, Comm. Anal. Geom. 6 (1998), 705-747.

[7] A. Strominger, S.T. Yau, and E. Zaslow, Mirror Symmetry is TDuality, Nucl. Phys. B479 (1996), 243-259. MR 97j:32022.

[8] S.T. YAU, On the Ricci curvature of a compact Kähler manifold and the complex Monge-Ampére equations. I, Comm. Pure Appl. Math. 31 (1978), 339-411. MR 81d:53045. 